\title{
ANÁLISIS BIBLIOMÉTRICO DE LA PRODUCCIÓN CIENTÍFICA SOBRE COVID-19 EN LATINOAMÉRICA
}

\section{BIBLIOMETRIC ANALYSIS OF SCIENTIFIC PRODUCTION ON COVID-19 IN LATIN} AMERICA

\section{Raúl José Martelo Gómez ${ }^{1}$ \\ Clara Judith Brito Carrillo² \\ David Antonio Franco Borré ${ }^{3}$}

Universidad de Cartagena

\section{RESUMEN}

El objetivo de esta investigación fue caracterizar la producción científica de países

$1 \quad$ Ingeniero de Sistemas, Especialista en Redes $y$ Telecomunicaciones, Magister en Informática. Docente del Programa de Ingeniería de Sistemas, Universidad de Cartagena, Cartagena de Indias, Colombia. Líder del Grupo de Investigación INGESINFO. ORCID: https://orcid.org/00000002-4951-0752. Mail: rmartelog1@unicartagena.edu.co. Teléfono: 3106359729.

2 Trabajadora Social, Especialista en Gerencia Social y Magister en Desarrollo y Gestión de Empresas Sociales. docente investigador asociado COLCIENCIAS. Docente de planta Universidad de La Guajira, Riohacha, Colombia. ORCID: https://orcid.org/0000-0001-8788-7326. Mail: clarabrito@uniguajira.edu.co.Teléfono: 3004933664.

$3 \quad$ Ingeniero de Sistemas, Magister en Ciencias Computacionales. Docente del Programa de Ingeniería de Sistemas, Universidad de Cartagena, Cartagena de Indias, Colombia. Líder del Grupo de Investigación GIMATICA. ORCID: https://orcid.org/0000-0001-7500-0206. Mail: dfrancob@unicartagena.edu.co. Teléfono: 3008143058. latinoamericanos sobre COVID-19 al 12 de julio del 2021. En cuanto a la metodología, se realizó un estudio bibliométrico en la base de datos SCOPUS. Se incluyeron artículos originales y originales breves en los que consignaron países latinoamericanos. Se describió la producción científica según el número de documentos, país, institución de procedencia, área temática, revistas científicas en las que se publicaron, el financiamiento de las investigaciones, y las redes de colaboración. Respecto a los resultados se incluyeron 10,697 artículos, de los cuales el 63.1 fueron artículos de investigación. Brasil fue el país más prolífico en producir investigación sobre Covid-19. De las cinco primeras revistas con mayor producción, dos fueron extranjeras y de las instituciones con mayor producción 
científica, la gran mayoría pertenecen al sector educación, liderando la Universidad de Sao Pablo. Teniendo en cuenta lo anterior, se concluyó que la mayoría de las publicaciones provenían de Brasil, mientras que Haití tuvo la menor contribución. Los artículos de revistas se reconocen como el principal método de difusión del conocimiento y la mayoría de estos artículos se publican entre las revistas más prestigiosas de Latinoamérica.

\section{PALABRAS CLAVE:}

Bibliometría, producción científica, infecciones por Coronavirus, pandemia, investigación científica.

\section{ABSTRACT}

The objective of this research was to characterize the scientific production of Latin American countries on COVID-19 as of July 12, 2021. Regarding the methodology, a bibliometric study was carried out in the SCOPUS database. Original and short original articles were included in which Latin American countries were consigned. Scientific production was described according to the number of documents, country, institution of origin, subject area, scientific journals in which they were published, research funding, and collaboration networks. Regarding the results, 10,697 articles were included, of which 63.1 were research articles. Brazil was the most prolific country to produce research on Covid-19. Of the first five magazines with the highest production, two were foreign and of the institutions with the highest scientific production, the vast majority belong to the education sector, led by the University of Sao Pablo. Taking into account the above, it was concluded that most of the publications came from Brazil, while Haiti had the least contribution. Journal articles are recognized as the main method of disseminating knowledge and most of these articles are published among the most prestigious magazines in Latin America.

\section{KEYWORDS}

Bibliometrics, scientific production, Coronavirus infections, pandemic, scientific research.

\section{INTRODUCCIÓN}

A mediados de diciembre de 2019 se detectó por primera vez en Wuhan, provincia de Hubei, China; un brote causado por el nuevo coronavirus humano del síndrome respiratorio agudo grave de tipo 2 (SARS-CoV-2), denominado COVID-19 (Abdullahi, et al., 2020). Sin duda alguna, se trata de una enfermedad altamente contagiosa (Bhadra, et al., 2021), lo cual se puede evidenciar en el rápido aumento del número de pacientes infectados por COVID-19 y la naturaleza altamente transmisible de la enfermedad (Das, et al., 2021). Tal es el caso, que para el 11 de marzo de 2020 la OMS declaró el estado de pandemia por SARS-CoV-2 tras la afectación de más de 125 países y con más de 130.000 casos confirmados en el mundo (Burgos, et al., 2020). Para el 17 de julio de 2020, ya se habían presentado más de 13 millones de casos confirmados y 580.000 muertes reportadas (Kim, 2020), y al 23 de mayo de 2021 un total de 65819095 de infectados acumulados y la muerte de más de 3 millones de personas fueron reportadas a nivel global (Seminario, 2021).

El hecho de que esta enfermedad se convirtiera en un fenómeno global e impredecible ha aumentado la sensación de incertidumbre en las personas en todo el mundo, haciendo que el brote sea aún más estresante que sus predecesores (Horesh \& Brown, 2020). En este sentido, la emergencia de salud pública, los impactos económicos (Maital \& Barzani, 2020), 
sociales (Prime, et al., 2020) y otros aspectos, de la pandemia en la vida de las personas en varios países crearon una catástrofe global, y aún se desconoce el alcance total de su impacto (Holmes, et al. 2020). Por lo anterior, este tema ha sido el foco principal de científicos e investigadores en los diferentes sectores en todo el mundo (Chahrour, et al., 2020). No obstante, el todavía en curso Covid-19 también ha afectado la forma en que se realizan, publican y difunden las investigaciones académicas (Fonkou, et al., 2021). Esto se evidencia principiante en el creciente número de publicaciones enfocadas en COVID-19 que se han publicado desde que se detectó por primera vez, así como en los nuevos conocimientos científicos que aportan información esencial con respecto tanto a la contención de la enfermedad como al tratamiento de los pacientes (Chahrour, et al., 2020).

En resumidas cuentas, el ámbito científico ha sufrido lo que podría llamarse una explosión de publicaciones relacionadas con diferentes aspectos de COVID-19y las revistas académicas son la fuente de medios más importante para la difusión de dichas investigaciones. Cabe señalar que existen varias revisiones y estudios bibliométricos que analizan datos mundiales (Dehghanbanadaki, et al., 2020), (Fan, et al., 2020), (Jacimovic, et al.,s.f.). Sin embargo, se conocen pocos estudios centrados en publicaciones científicas en América Latina, y mucho menos en el amplio campo de la salud. Por lo anterior, el presente estudio tuvo como objetivo arrojar luz sobre el estado actual de los documentos publicados sobre Covid-19 en Latinoamérica. Elanálisiscentradoendescribirlas revistas más productivas, instituciones y países, financiamiento $t$ también se muestra un mapa de visualización en red realizado en VOSviewer. Se examinaron 10,697 publicaciones producto de investigaciones científicas sobre Covid-19 en países de Latinoamérica recuperados de la base de datos SCOPUS comprendidos entre 2019 y a mediados del mes de julio de 2021, teniendo en cuenta indicadores de cantidad y calidad.

\section{METODOLOGÍA}

\section{Diseño y población de estudio}

Se realizó una búsqueda bibliométrica basada en una adaptación al diseño metodológico propuesto por Pineda (2015), el cual se compone de 3 fases; establecer la ecuación de búsqueda y su resultado, desarrollar el ejercicio bibliométrico obteniendo como resultado el análisis bibliométrico para la recolección de los datos y finalmente la última fase busca consolidar información para su difusión y se obtiene como resultado el resumen de los datos y establecimiento de los principales hallazgos de la búsqueda. En función de lo planteado, en este documento se exponen los resultados de realizar un análisis bibliométrico de la producción científica sobre COVID-19, para obtener una visión general de los avances de la investigación acerca de este tema. El desarrollo de un análisis bibliométrico implica la medición de la literatura desde varios aspectos (CastilloMuñoz, et al., 2017). Entre los cuales se analiza y mide la calidad y cantidad de publicaciones, con la aplicación de métodos estadísticos y matemáticos, para identificar tendencias relacionadas con avances y desarrollos científicos (Van, 2005).

\section{Variables y estrategia de búsqueda}

En el análisis bibliométrico se pueden utilizar tres tipos de indicadores, el que indica la cantidad (está relacionado con la medición de la productividad de publicaciones, en términos de números), el relacionado con la calidad (mide el impacto de las publicaciones en relación con las citas recibidas y finalmente) y el indicador de 
estructura (mide la relación entre publicaciones) (Cadavid-Higuita, et al., 2012). En el análisis de la presente investigación, se utilizan los tres indicadores, con el fin de obtener una visión global de la producción científica sobre la enfermedad COVID-19 ocasionada por el nuevo coronavirus o Sars Cov 2. En este sentido, como fuente informativa se seleccionaron los datos arrojados por una ecuación de búsqueda en la base de datos de SCOPUS.

Los datos obtenidos, fueron artículos en los cuales, en su resumen, titulo, o palabras clave, contenían las palabras COVID-19, SARSCoV-2o 2019-nCoV y se utilizó la ecuación de búsqueda TITLE-ABS-KEY ( covid-19 OR sarscov-2 OR 2019-ncov ) limitada a los años 2019, 2020 y 2021 y limitada a los países de Latinoamérica: Brasil, México, Colombia, Chile, Argentina, Perú, Ecuador, Cuba, Venezuela, Uruguay, Costa Rica, Bolivia, Honduras, Panamá, Paraguay, Guatemala, República Dominicana, Puerto Rico,

El Salvador, Nicaragua y Haití. se obtuvieron 10,697 documentos.

\section{Indicadores bibliométricos}

La información obtenida de la base de datos Scopus incluyó: número de publicaciones por año, países activos, revistas activas, instituciones activas e información de citas. Los mismos datos fueron exportados al software Excel para tabularlos o mapearlos.

\section{Visualización}

Los datos obtenidos para el COVID-19 se exportaron al programa VOSviewer con fines cartográficos (Van \& Waltman, 2021). Se realizó un mapeo para las palabras que se encuentran con más frecuencia en los títulos / resúmenes de los documentos recuperados y para los países con una contribución mínima de 20 documentos para visualizar la colaboración internacional en investigación sobre el tema en estudio.

\section{RESULTADOS}

La consulta de búsqueda encontró 10697 documentos relacionados con la enfermedad COVID-19, publicados desde 2019 hasta el 12 de julio de 2021 según la base de datos SCOPUS. La mayoría de los documentos fueron artículos de investigación $(n=6747$; $63.1 \%$ ) seguidos de artículos de revisión (n $=1358 ; 12,7 \%)$. El Gráfico de crecimiento anual de las publicaciones mostró un fuerte crecimiento (Figura 1). El número de publicaciones en 2020 presentó un incremento de aproximadamente 99.58\% con respecto al 2019. De las publicaciones obtenidas, los tres patrocinadores de financiación más activos fueron: Consejo Nacional de Desenvolvimiento Científico y Tecnológico $(n=770)$, Ministerio da Ciencia, Tecnología e Innovación ( $\mathrm{n}=679)$ y Coordinación para la Perfeccionamiento del Personal de Educación Superior $(n=652)$. 
Figura 1. Crecimiento anual de publicaciones relacionadas con COVID-19 (2019-2021)

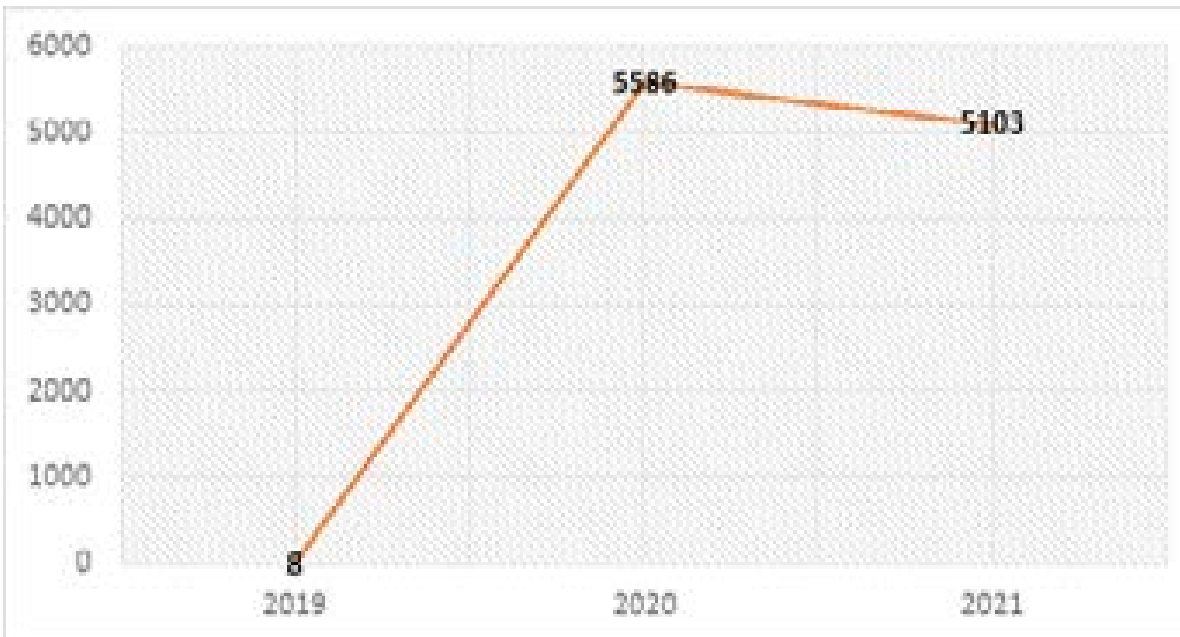

Fuente: Elaboración propia con base en datos de Scopus

El gráfico muestra que para el año 2019 se registraron 8 documentos. Para el año 2020 se encontraron 5590 documentos referentes a la enfermedad de COVID-19. Para el 2021 hasta el 12 de julio se evidenciaron 5103 documentos, lo cual indica que cada vez se avanza en investigación acerca de esta enfermedad. Por otro lado, los documentos obtenidos fueron publicados en revistas pertenecientes a diferentes áreas temáticas. Aproximadamente el $42.9 \%$ de los documentos se publicaron en revistas del área temática de medicina, mientras que un $20.2 \%$ fueron publicados en otras áreas temáticas. Un $8.9 \%$ en el área de las ciencias sociales y las áreas temáticas de Bioquímica, Genética y Biología Molecular e Inmunología y microbiología, ocuparon el tercero y cuarto lugar con un $6.1 \%$ y $4.7 \%$ respectivamente.

En cuanto a la distribución geográfica de los documentos obtenidos en la búsqueda, como lo muestra la Figura 2, el país latinoamericano con

Figura 2. Número de publicaciones sobre COVID-19 en diferentes países de Latinoamérica

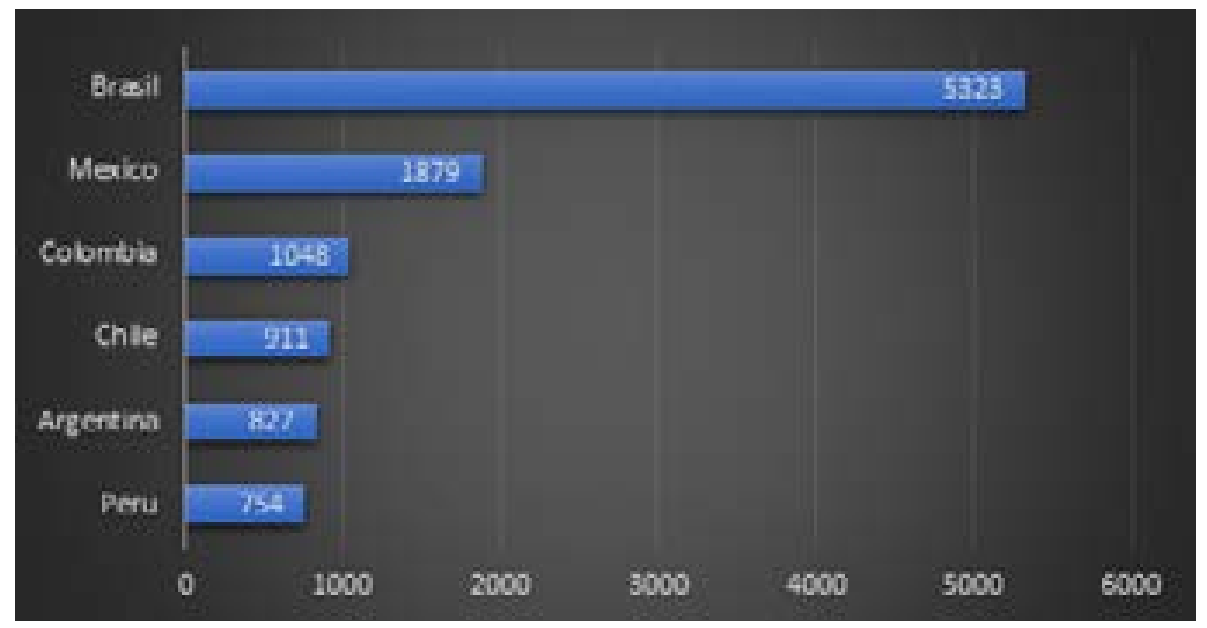

Fuente: Elaboración propia con base en datos de Scopus 
Figura 3. Crecimiento anual de publicaciones relacionadas con COVID-19 en países de Latinoamérica.

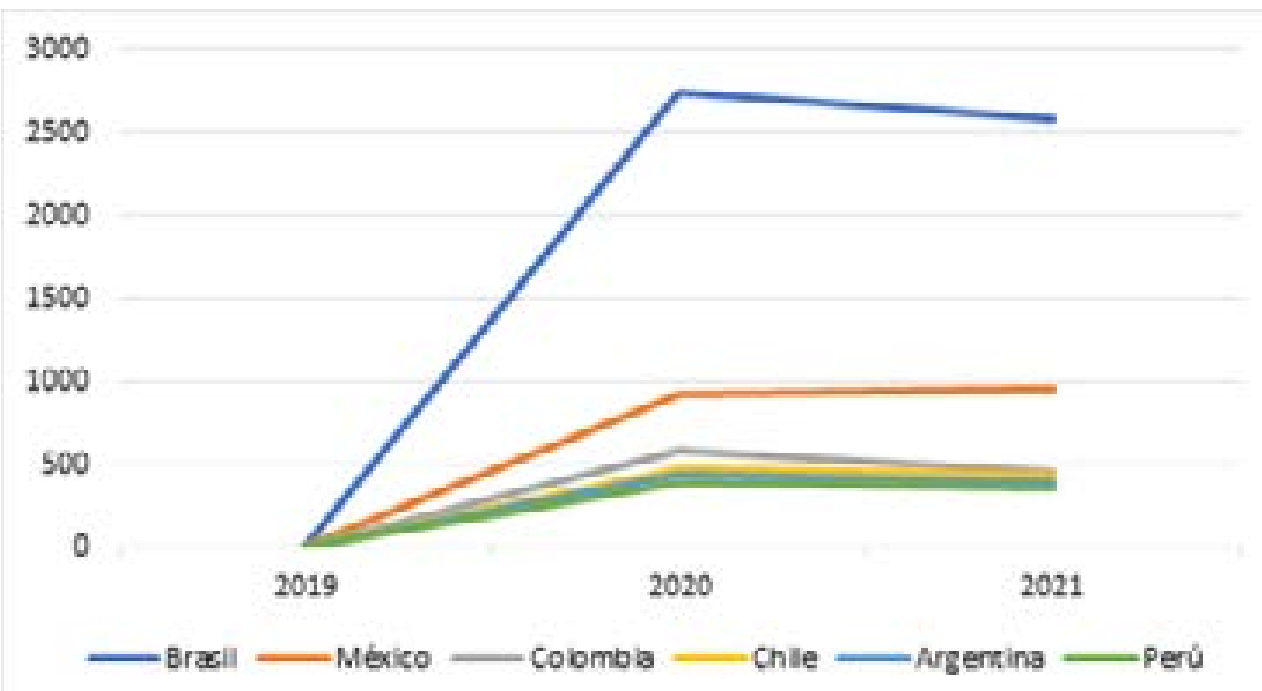

Fuente: Elaboración propia con base en datos de Scopus

El crecimiento anual en los seis países con mayor contribución en Latinoamérica mostró un patrón similar con un papel de liderazgo para Brasil quien en el 2019 cerró con 2742 documentos y hasta inicios del mes de julio de 2021 contaba con 2577 productos.

Con respecto a las revistas activas, en la Figura
4 se muestran las cinco revistas más prolíficas. Como se observa, la que ocupa el primer lugar es Plos One con 136 publicaciones seguida de International Journal of Environmental Research and Public Health con 122 publicaciones y en el tercer lugar se encuentra Cadernos De Saude Publica con 101 documentos publicados sobre COVID-19.

Figura 4. Las cinco principales revistas activas en la publicación de investigación sobre COVID-19

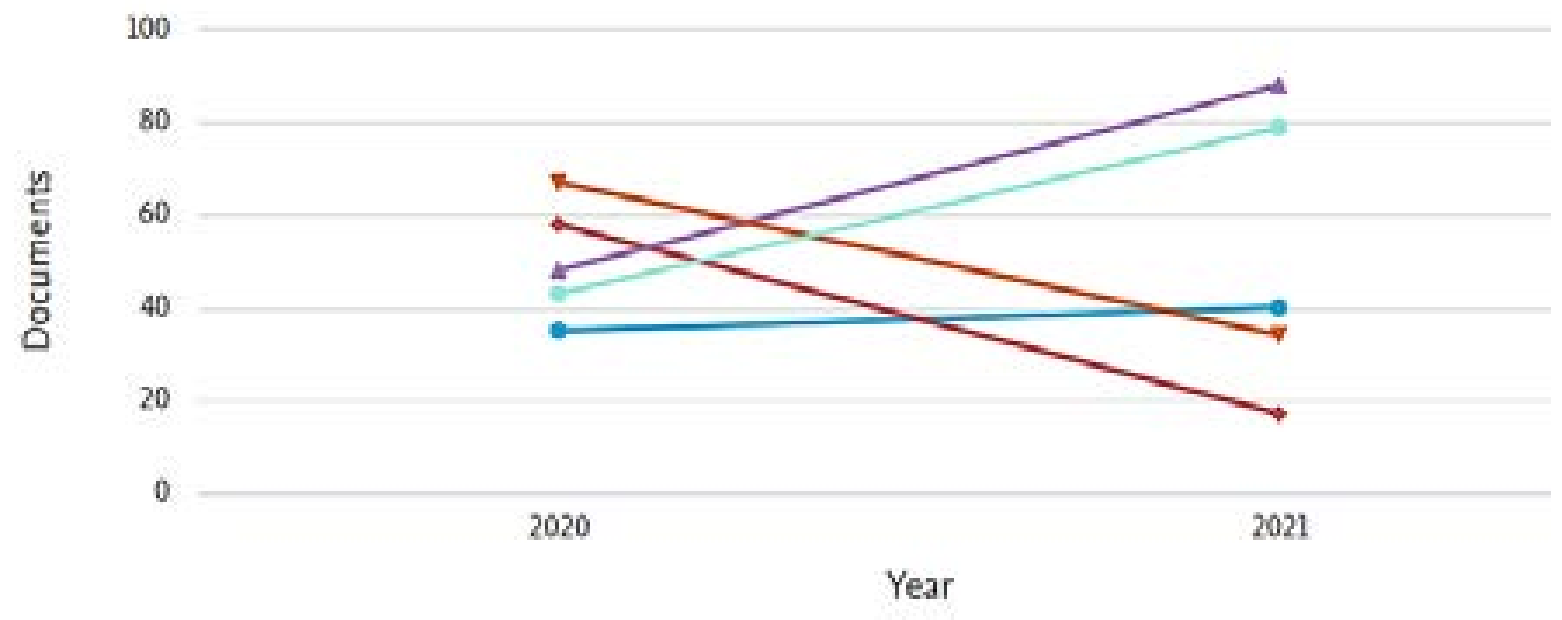

Revista Brasileira De Enfermagem $\neq$ Ciencia E Saude Coletiva $\neq$ Plos One * Cadernos De Saude Publica International journal of Ervironmental Research And Public Health 
La Tabla 1 muestra seis columnas, la primera corresponde al nombre de la fuente o revista en las cuales se ha publicado con mayor frecuencia sobre COVID-19, la segunda corresponde al país, la tercera, al número de documentos (D), la cuarta al índice h, la quinta columna, corresponde al índice de impacto (SJR), el cual mide la influencia o prestigio científico de las revistas mediante el análisis de la cantidad y la procedencia de las citas que recibe una revista científica (Bencomo, et al., 2014). Por último, la columna que corresponde al cuartil SClmago, el cual es un indicador que permite evaluar la importancia relativa de una revista dentro del total de revistas de su área.

Tabla 1. Las cinco principales revistas activas en la publicación de investigaciones relacionadas con los COVID-19 (2019-2021)

\begin{tabular}{|l|l|l|l|l|l|}
\hline Source & Country & D & h-index & SJR & $\begin{array}{l}\text { SCImago } \\
\text { Quartile }\end{array}$ \\
\hline Plos One & Estados Unidos & 136 & 332 & 0.990 & Q1 \\
\hline $\begin{array}{l}\text { International Journal of Environmental } \\
\text { Research and Public Health }\end{array}$ & Suiza & 122 & 113 & 0.747 & Q2 \\
\hline Cadernos De Saude Publica & Brasil & 101 & 77 & 0.633 & Q2 \\
\hline Ciencia E Saude Coletiva & Brasil & 75 & 46 & 0.702 & Q2 \\
\hline Revista Brasileira De Enfermagem & Brasil & 75 & 21 & 0.274 & Q3 \\
\hline
\end{tabular}

Fuente: Elaboración propia con base en datos de Scopus y SCImago

Se observa que la revista con mayor número de publicaciones, es Plos One de Estados Unidos, la cual tiene el SJR más alto (0.99), el índice $\mathrm{h}$ más alto (332) y se encuentra en el cuartil Q1, lo anterior evidencia el gran impacto que genera esta revista. De igual manera, se observa que las siguientes tres revistas se encuentran en el cuartil Q2, demostrando que están dentro del conjunto compuesto por el $25 \%$ de las revistas con más alto impacto y en el quinto lugar se encuentra Revista Brasileira De Enfermagem, es una revista de Brasil con 75 publicaciones, índice h 21, un SJR de 0.274 perteneciente al cuartil Q3.

En lo que respecta a las instituciones activas, la Figura 5 muestra las diez principales instituciones activas basada en la afiliación de los autores, el cual indica que la Universidad de Sao Paulo fue la institución más activa en la publicación de documentos vinculados al COVID-19 ( $n=$ 1217), seguida de la Fundación Oswaldo Cruz $(n=424)$, y la Universidad de Federal de Sao Paulo $(n=394)$. 
Figura 5. Las diez principales instituciones activas en la publicación de investigaciones relacionadas con COVID-19 (2019-2021)

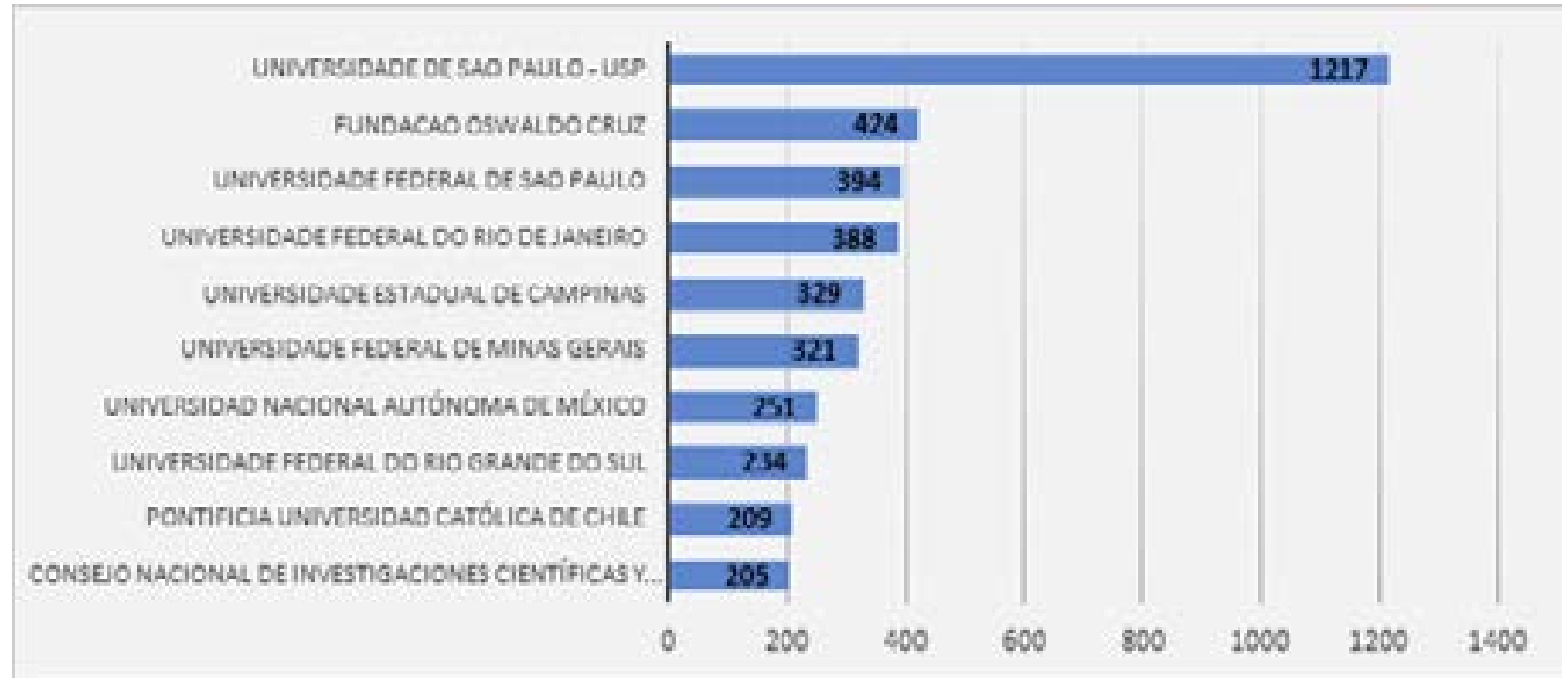

Fuente: Elaboración propia con base en datos de Scopus

Por otro lado, teniendo en cuenta que la unidad básica de los artículos son las palabras, en la Figura 6 se presenta la visualización de la red de los términos en los títulos y resúmenes de publicaciones relacionadas mostró siete grupos distintos, no obstante, sobresalen tres grupos que representan a tres temas principales de investigación: Tiempo / Pandemia (color rojo), Sars Cov / Infección (color amarillo), y Paciente (color azul).

Figura 6. Mapa de visualización en red de publicaciones relacionadas con COVID-19.

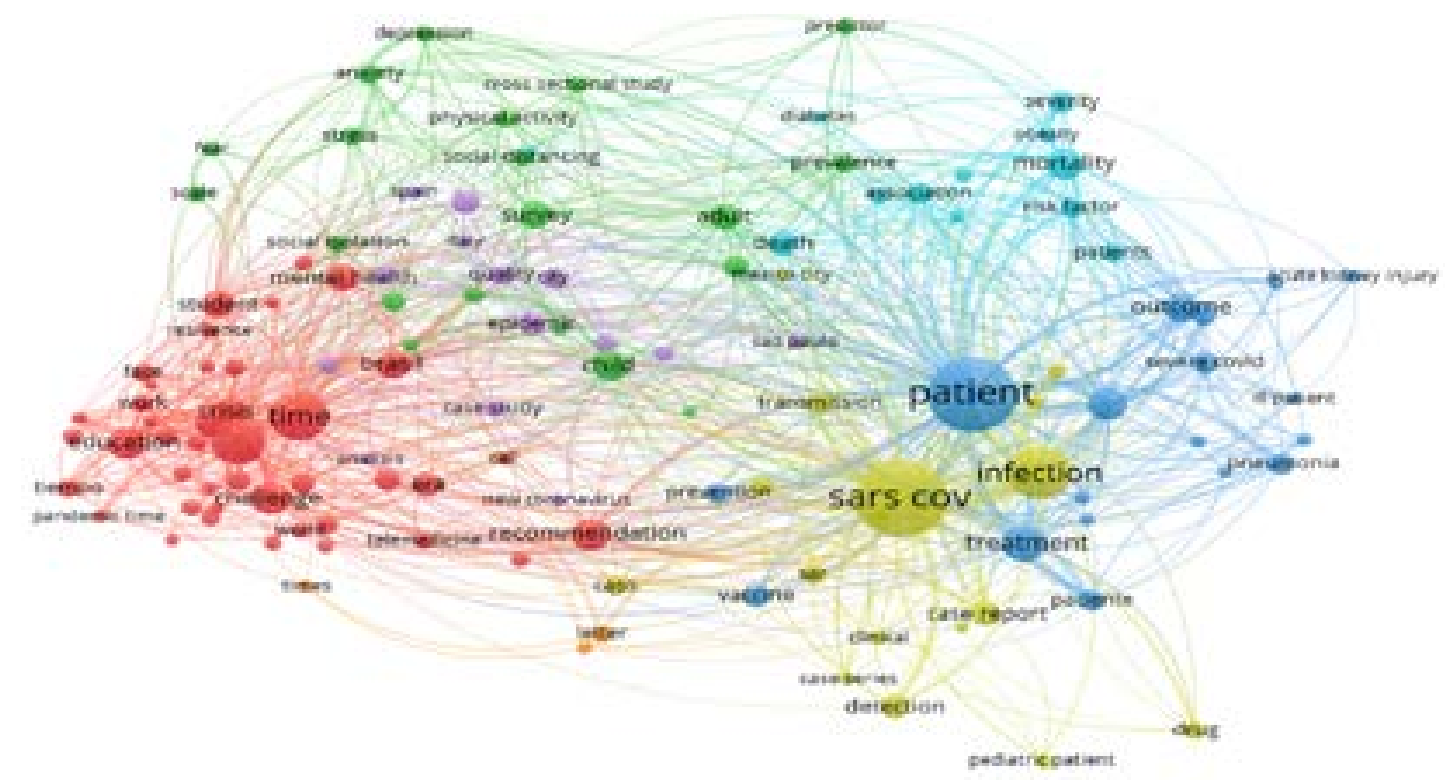




\section{DISCUSIÓN}

El análisis bibliométrico es cada vez más utilizado para la revisión de tendencias y avances en diferentes campos y áreas de investigación. El presente estudio se realizó con el fin de evaluar y analizar publicaciones científicas relacionadas con la enfermedad de COVID-19 en cualquier parte del artículo, asumiendo que dichas publicaciones representan investigaciones relacionadas con uno o más aspectos de esta enfermedad.Elanálisisactualdedatosrepresenta diferentes dimensiones de la investigación de COVID-19, que incluye los principales países de Latinoamérica, Instituciones académicas y revistas que producen publicaciones sobre COVID-19. De estos países, Brasil. Es el país número uno en términos de investigación sobre COVID-19, seguido de México y, de manera distante, por países como Colombia, Chile, Argentina y Perú.

Se evidenció un fuerte aumento en el número de publicaciones reflejando un creciente interés de los investigadores en temas relacionados, esta tendencia puede que se prolongue más de lo esperado, debido a que el Covid-19 como un virus muy contagioso y de rápida propagación, podría despertar más la atención. De igual manera, este estudio también evidenció que la mayoría de las investigaciones fueron asociadas a la medicina y a las ciencias sociales. Sin embargo, el $20 \%$ de los documentos recuperados estaban asociados con otras disciplinas.

La mayoría de las revistas activas estaban en el rango $Q 1$ y $Q 2$, lo cual sugiere que el contenido de la investigación y la calidad de las publicaciones relacionadas con el tema en estudio, son elevados y beneficiosos para el personal interesado. Asimismo, el estudio actual indicó que la revista PloS One ocupó el primer lugar, seguida de cerca por International Journal of Environmental Research and Public Health con 14 documentos de diferencia. De igual manera, el hallazgo con respecto a la lista de las
5 principales revistas activas está de acuerdo con los hallazgos de un artículo reciente sobre la producción científica sobres Covid-19 a nivel mundial (Farooq, et al., 2121).

Las universidades y los académicos están en condiciones de interactuar y tener un papel de liderazgo a través de la colaboración en la investigación sobre Covid-19. En este sentido, el hallazgo de que nueve de las diez principales instituciones activas son instituciones académicas refleja el creciente papel de los académicos en la investigación sobre Covid-19. En cuanto a la financiación de la investigación relacionada con Covid-19, el más activo fue el Consejo Nacional de Desenvolvimiento Científico y Tecnológico, el cual es un órgano del Ministerio de Ciencia, Tecnología e Innovación de Brasil para promover la investigación, cuyo objetivo inicial fue conseguir preparar a Brasil para el dominio de la energía atómica, tema de importancia estratégica a medios del siglo $\mathrm{XX}$. Con el paso del tiempo su papel fue ampliándose a otras áreas de conocimiento. La financiación es una fuerza impulsora importante para las publicaciones, el crecimiento de la colaboración científica y un mayor impacto de los artículos científicos (Gush, et al., 2018).

\section{LIMITACIONES}

El presente estudio tiene algunas limitaciones que se presentan a continuación. En primer lugar, no se incluyó artículos publicados en bases de datos ajenas a Scopus, lo cual afectó negativamente el número de publicaciones recuperadas de países / regiones con revistas locales o regionales que no están indexadas en Scopus. En segundo lugar, el estudio actual se centró en la literatura revisada por pares y no incluyó literatura gris. A veces, la literatura gris incluye información importante y evaluaciones del progreso del Covid-19. En tercer lugar, se incluyeron en el análisis todo tipo de publicaciones, como notas, cartas, reseñas y editoriales. Sin embargo, estos tipos pueden no 
representar una contribución de investigación original. A pesar de todo esto, el estudio actual proporciona una imagen completa de la productividad Latinoamérica de la investigación relacionada con Covid-19 y las áreas temáticas de interés para los investigadores de todo el mundo.

\section{CONCLUSIÓN}

Las publicaciones relacionadas con la investigación de COVID-19 han crecido rápidamente desde que surgió la enfermedad. Al mostrar el estado actual de la literatura a través de un análisis de las publicaciones encontradas en revistas indexadas a scopus, se proporciona información significativa para los investigadores e interesados en el tema. Los resultados de este documento de revisión bibliométrica evidencian que la literatura relacionada con Covid-19 publicada en revistas revisadas por pares ha crecido recientemente con un énfasis en temas relacionado con la psicología, vacuna y secuelas causadas por esta enfermedad. La mayoría de las publicaciones provenían de Brasil, mientras que Haití tuvo la menor contribución. Los artículos de revistas se reconocen como el principal método de difusión del conocimiento y la mayoría de estos artículos se publican entre las revistas más prestigiosas de Latinoamérica.

\section{REFERENCIAS BIBLIOGRÁFICAS}

Burgos, F., Martínez, J., \& Cordovilla, R. (2020). Impact of the COVID-19 Pandemic on Lung Function Laboratories: Considerations for "Today" and the "Day After. Elsevier, 611-612. doi:10.1016/j. arbres.2020.07.001

Abdullahi, I., Uchenna, A., Mustapha, J., Fasogbon, S., Bassey, I., Sikiru, I., . . . Nwofe, J. (2020). Exploring the genetics, ecology of SARS-COV-2 and climatic factors as possible control strategies against COVID-19. Le Infezioni in Medicina, 28(2), 166-173.

Bencomo, D., Sánchez, S., Hernández, K., Cárdenas, L., Fundora, J., \& Dorta, A. (2014). Herramientas para medir la eficacia de la Universidad de Ciencias Médicas de La Habana: SCImago Institution Rankings 2010-2012. Revista Habanera de Ciencias Médicas, 13(2), 291-301.

Bhadra, A., Mukherjee, A., \& Sarkar, K. (2021). Impact of population density on Covid-19 infected and mortality rate in India. Modeling Earth Systems and Environment, 7(1), 623-629.

Cadavid-Higuita, L., Awad, G., \& FrancoCardona, C. (2012). A bibliometric analysis of a modeled field for disseminating innovation. Estudios Gerenciales, 213-236.

Castillo-Muñoz, C., Ripoll, V., \& Urquidi, A. (2017). Revelaciones voluntarias sobre Responsabilidad Social Corporativa (RSC): Un análisis bibliométrico y sistémico. Revista Espacios, 38(26).

Chahrour, M., Assi, S., Bejjani, M., Nasrallah, A., Salhab, H., Fares, M., \& Khachfe, H. (2020). A bibliometric analysis of COVID-19 research activity: a call for increased output. . Cureus, 12(3).

Das, A., Islam, N., Billah, M., \& Sarker, A. (2021). COVID-19 pandemic and healthcare solid waste management strategy-A mini-review. Science of The Total Environment, 778. doi:https://doi. org/10.1016/j.scitotenv.2021.146220

Dehghanbanadaki, H., Seif, F., Vahidi, Y., Razi, F., Hashemi, E., Khoshmirsafa, M., \& Aazami, H. (2020). Bibliometric analysis of global scientific research 
on Coronavirus (COVID-19). . Medical Journal of the Islamic Republic of Iran, 34(51). doi:https://doi.org/10.7759/ cureus.7357

Fan, J., Gao, Y., Zhao, N., Dai, R., Zhang, H., Feng, X., . . Shisan, B. (2020). Bibliometric Analysis on COVID-19: A Comparison of Research Between English and Chinese Studies. Frontiers in public health, 8(477).

Farooq, R., Rehman, S., Ashiq, M., Siddique, N., \& Ahmad, S. (2121). Bibliometric analysis of coronavirus disease (COVID-19) literature published in Web of Science 2019-2020. Journal of Family \& Community Medicine, 28(1).

Fonkou, M., Bragazzi, N., Tsinda, E., Bouba, Y., Mmbando, G., \& Kong, J. (Julio de 2021). Covid-19 pandemic related research in africa: Bibliometric analysis of scholarly output, collaborations and scientific leadership. International Journal of Environmental Research and Public Health, 18(14).

Gush, J., Jaffe, A., Larsen, V., \& Laws, A. (2018). The effect of public funding on research output: the New Zealand Marsden Fund. New Zealand Economic Papers, 52(2), 227-248.

Holmes, E., O'Connor, R., Perry, V., Tracey, I., Wessely, S., Arseneault , L., . . . Bullmore, E. (2020). Multidisciplinary research priorities for the COVID-19 pandemic: a call for action for mental health science. The Lancet Psychiatry, 366(20), 1- 14.

Horesh, D., \& Brown, A. (2020). Covid-19 response: Traumatic stress in the age of Covid-19: A call to close critical gaps and adapt to new realities. Psychological Trauma: Theory, Research, Practice, and Policy, 12(4), 331- 335.

Jacimovic, J., Jakovljevic, A., Nagendrababu, V., Duncan, H., \& Dummer, P. (s.f.). A bibliometric analysis of the dental scientific literature on COVID-19. Clinical oral investigations. doi:https:// doi.org/10.1007/s00784-021-03916-6

Kim, J. (2020). ¿ Cómo controló Corea del Sur el Covid-19? Política exterior,, 34(195), 126-134.

Maital, S., \& Barzani, E. (2020). The global economic impact of COVID-19: A summary of research. Samuel Neaman Institute for National Policy Research, 1-12.

Pineda, D. (2015). Análisis bibliométrico para la identificación de factores de innovación en la industria alimenticia. $A D$-Minister, 95-126.

Prime, H., Wade, M., \& Browne, D. (2020). Risk and resilience in family well-being during the COVID-19 pandemic. American Psychologist, 75(5), 631- 643.

Seminario, R. (2021). La pandemia de la COVID-19 y su impacto económico, social y salud. Revista Científica Ágora, 8(1).

Van, A. (2005). Fatal attraction: Conceptual and methodological problems in the ranking of universities by. Scientometrics, 62(1), 133-143.

Van, E., \& Waltman, L. (2021). Text mining and visualization using VOSviewer. Recuperado el 7 de Julio de 2021, de ext mining and visualization using VOSviewer: https://arxiv.org/ abs/1109.2058 